Case Report

\title{
Safety of Low Flow Sevoflurane Anesthesia for Patient with Dextrocardia and Situs Inversus Totalis: A Case Report
}

\author{
Agreta Gecaj- Gashi ${ }^{1}$, Shaqir Uka ${ }^{1}$, Zorica Nikolova ${ }^{2}$, Aurora Bakalli ${ }^{3}$ and Sami Salihu ${ }^{4}$ \\ ${ }^{1}$ Clinic of Anesthesiology \& Intensive Care, University Clinical Centre of Kosova, Prishtina, \\ Republic of Kosova \\ ${ }^{2}$ Clinic of Anesthesiology \& Intensive Care, University Clinical Center of Skopje-Macedonia \\ ${ }^{3}$ Department of Cardiology, Clinic of Internal Medicine, University Clinical Center of Kosova Prishtina, \\ Republic of Kosova \\ ${ }^{4}$ Clinic of Maxillofacial Surgery, University Clinical Centre of Kosova, Prishtina, Republic of Kosova \\ Correspondence should be addressed to: Agreta Gecaj-Gashi; agretag@yahoo.com
}

Received date: 9 September 2013; Accepted date: 29 October 2013; Published date: 20 December 2013

Academic Editor: Somboon Thienthong

Copyright (C) 2013 Agreta Gecaj- Gashi, Shaqir Uka, Zorica Nikolova, Aurora Bakalli and Sami Salihu. Distributed under Creative Commons CC-BY 3.0

\begin{abstract}
Introduction: Situs inversus totalis with dextrocardia also named situs inversus totalis is a rare congenital condition in which the anatomy of the abdominal and thoracic organs are arranged in a mirror image reversal of the normal positioning. Case Report: A 42-year-old Albanian female was scheduled for elective maxillofacial surgery, cyst in the left side of the neck, and previously undiagnosed dextrocardia with situs inversus was picked up in preanesthetic evaluation by an anesthesiologist, during auscultation of the heart revealed a grade 2/6 holosystolic murmur at the left parasternal border. General anesthesia was induced with propofol $3 \mathrm{mg} / \mathrm{kg}$, fentanyl $3 \mathrm{mcg} \mathrm{kg}$, rocuronium $1 \mathrm{mg} / \mathrm{kg}$ to facilitate endotracheal intubation and maintained with sevoflurane 1.0- 1, 5 MAC, in $02 / \mathrm{N} 20$ 50:50\%. After tracheal intubation, the fresh gas flow was set to 4 L.min ${ }^{-1}\left(\mathrm{O}_{2} 2\right.$ L.min, $\mathrm{N}_{2} \mathrm{O} 2$ L.min). When the target gas concentrations have been achieved, FGF was reduced in $1 \mathrm{l} / \mathrm{min}$, with the relative proportion of $02 / \mathrm{N} 20$ titrated to maintain the inspired oxygen fraction $\left(\mathrm{FiO}_{2}\right)$ between 0.30 and 0.35 . Inhaled and exhaled gas concentrations were continuously monitored. The patient was calm after extubation (score 1-of agitation), awareness according to Awareness Categorization was pointed with score 1- no awareness. In the PACU she was hemodynamically stable and after full recovery returned to the ward. Results of arterial blood gas analyzes BUN, AST, ALT, LDH, glycemia, total bilirubin, proteinogram, haemogram and electrolytes were within normal reference range. Her postoperative course was uneventful and she was discharged on the third postoperative day. Conclusion: With particular attention in preanesthetic visit we managed to find out dextrocardia with situs inversus totalis. We realized low flow sevoflurane anesthesia, which showed to be safe.
\end{abstract}

Keywords: Dextrocardia, Low flow anesthesia, situs inversus, sevoflurane.

Cite this Article as: Agreta Gecaj- Gashi, Shaqir Uka, Zorica Nikolova, Aurora Bakalli and Sami Salihu (2014)," Safety of Low Flow Sevoflurane Anesthesia for Patient with Dextrocardia and Situs Inversus Totalis: A Case Report", International Journal of Case Reports in Medicine, Vol. 2014 (2014), Article ID 574547, 


\section{Introduction}

Situs inversus totalis with dextrocardia (also named situs inversus totalis) is a rare congenital condition in which the anatomy of the abdominal and the thoracic organs are arranged in a mirror image reversal of the normal positioning. Situs inversus with dextrocardia is an autosomal recessive disease and it was first reported by the anatomist Hieronymus Fabricius in 1606 [cited in Cleveland 1926] [1]. The prevalence of dextrocardia with situs inversus is estimated at $2: 10,000,20 \%$ of such individuals have Kartagener's syndrome [2].

Although this rare condition is well described by a few medical and surgical journals, the anesthetic implications have not been clarified enough $[3,4]$.

Here we describe a case discovered with dextrocardia and situs inversus totalis, application and safety of low flow sevoflurane anesthesia in this patient.

\section{Case Presentation}

A 42 year old Albanian female, ASA II, weight $68 \mathrm{~kg}$, was scheduled for elective maxillofacial surgery for cystic formation in the left side of the neck, with previously undiagnosed dextrocardia with situs inversus, was picked up in preanesthetic evaluation by anesthesiologist, during an auscultation of the heart revealed a grade $2 / 6$ holosystolic murmurs at the left parasternal border. Vesicular breath sounds without crackles or wheeze were heard on chest auscultation. Her skin and mucosa's was lightly pale, but with normal profile of laboratory investigations, a HR (heart rate) of $70 / \mathrm{min}$ and BP (blood pressure) of $125 / 75 \mathrm{mmHg}$.

The airway exam revealed no apparent abnormalities with Mallampatti Class 1 and good range of motion of the neck. There were no apparent deformities on examination. Her medical history does not indicate any previous health problems; she has had four normal vaginal deliveries without any complications.

Posteroanterior chest radiography was not labeled in right or left side. So, we asked for a repeated radiography with supervision or special instructions to verify correct leftsided and right- sided labeling. The second chest radiography revealed dextrocardia, with the cardiac apex to the right and the right- sided aortic arch associated with slight deviation of the trachea to the left and stomach bubble located on the right as well, but there was no evidence of bronchiectasis (see image below).

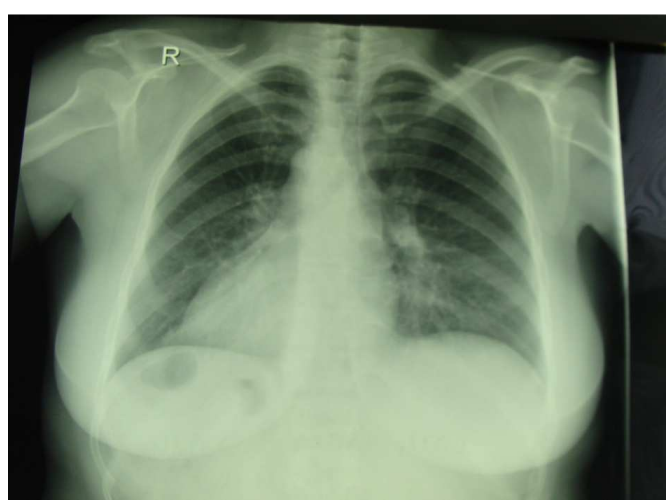

Figure 1: Chest radiography

We also asked for an ECG and a consultation from a cardiologist, who in echocardiography revealed dextrocardia with tricuspid regurgitation gr. I- II, the ejection fraction of $60 \%$, mild enlargement of the left atrium, mild hypertrophy of the interventricular septum and normal systolic function.

We then asked for thoracic and abdominal MRI, which found the dextrocardia with situs inversus totalis (see image below). 


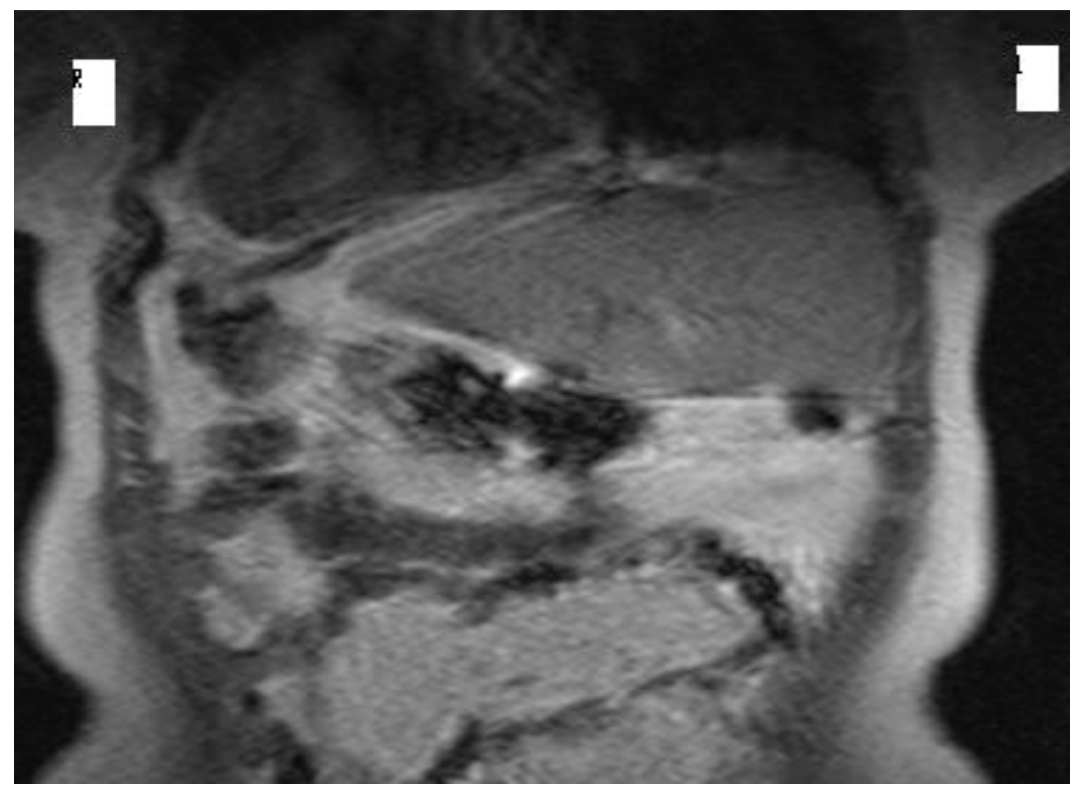

Figure 2: Abdominal MRI

\section{Anesthetic Management}

An 18 gauge intravenous line was placed and patient was premedicated with midazolam 1 mg IV (intravenous), 30 minutes before induction. In the operating room the patient was preoxygenated with oxygen $100 \%$ fresh gas flow $8 \mathrm{l} / \mathrm{min}$ for $3 \mathrm{~min}$, with routine monitoring: pulse oximetry, electrocardiogram leads were placed on exactly opposite spots to what is done for levocardia, arterial blood pressure was enhanced by performing a left radial arterial line.

General anesthesia was induced with propofol 3mg/kg, fentanyl $3 \mathrm{mcg} \mathrm{kg}$, rocuronium $1 \mathrm{mg} / \mathrm{kg}$ to facilitate endotracheal intubation and maintained with sevoflurane 1.0-1.5 MAC (Minimum Alveolar Concentration), $\quad \mathrm{O}_{2} / \mathrm{N}_{2} \mathrm{O} \quad 50: 50 \%$. The patient's trachea was intubated and her lungs were mechanically ventilated using PCV (Pressure Controlled Ventilation) mode
(Dragger, Fabius GS). After tracheal intubation, the fresh gas flow was set to 4 L.min ${ }^{-1}\left(\mathrm{O}_{2} 2\right.$ L.min ${ }^{-1}, \mathrm{~N}_{2} \mathrm{O} 2$ L.min $\left.{ }^{-1}\right)$, whereas sevoflurane 1.0-1.5 MAC for $10 \mathrm{~min}$. After the high flow phase, respectively when the target gas concentrations have been achieved, FGF (Fresh Gas Flow) was reduced in $1 \mathrm{l} / \mathrm{min}$, with the relative proportion of oxygen and nitrous oxide titrated to maintain the inspired oxygen fraction $\left(\mathrm{FiO}_{2}\right)$ between 0.30 and 0.35 . With the gas analyzer continuously monitoring inhaled and exhaled gas concentrations $\quad \mathrm{O}_{2}, \mathrm{~N}_{2} \mathrm{O}, \mathrm{CO}_{2}$, sevoflurane).

Ventilation was adjusted to maintain normal arterial oxygenation and end tidal $\mathrm{CO}_{2}$ at range $30-45 \mathrm{mmHg}$ during anesthesia. Analgesia was maintained with incremental doses of fentanyl whereas for neuromuscular block we used atracurium when required. Hemodynamic monitoring was continuously performed during the course of anesthesia, 
and the patient was hemodynamically stable perioperatively.

Reversal of muscle relaxation was achieved with IV administration of atropine/neostigmine in $0.5 / 2.5 \mathrm{mg}$. At the end of anesthesia, high $\mathrm{FGF} 4 \mathrm{l} / \mathrm{min} \mathrm{O}_{2}$ was administered to facilitate the washout of anesthetic agent from the patient, and to remove the agent from the circuit to the scavenging system.

After surgery, which lasted 75 minutes, extubation was smooth and the patient was transferred to PACU (Post-anesthesia Care Unit). The quality of recovery from anesthetics was assessed by recording the presence or absence of emergence agitation in the PACU [5]. The degree of agitation was assessed on a three-point scale: 1- calm, 2agitated but consolable, 3- severely agitated, inconsolable. Whereas, the recovery was assessed by using the Steward recovery score [6]. The patient was interviewed postoperatively for awareness also in the postanesthesia care unit (PACU) with the structured interview, modified from Brice et al., [7] and categorized with Awareness Categorization $[8,9]$.

Arterial blood samples were taken from radial artery and were analyzed immediately after sampling in an automatic blood gas analyzer to evaluate the impact of LFA (Low Flow Anesthesia) in respiratory gas exchange, for $\mathrm{PaO}_{2}, \mathrm{PaCO}_{2}$, and $\mathrm{pH}$. Measurements were performed at the induction of anesthesia, $30 \mathrm{~min}$ after beginning of low flow phase and 2 hours postoperatively. Venous blood samples were taken before induction, 2 hours postoperatively and in third day postoperatively, for measurement of blood urea nitrogen (BUN), serum creatinine, aspartate aminotransferase (AST), alanine aminotransferase (ALT), lactate dehydrogenase (LDH), alkaline phosphatase (ALP) total bilirubin and protein, albumin, glycemia, basic hematology (hemoglobin, packed cell volume, platelet count, WBC count) and electrolytes (serum $\mathrm{K}, \mathrm{Na}$ and $\mathrm{Ca}$ ).
Patient was calm after extubation (score 1-of agitation), the recovery was smooth with simplified Post -Anesthetic Recovery Score 6. Awareness according to Awareness Categorization was pointed with score 1- no awareness: no reported awareness or a vague description.

In the PACU she was hemodynamically stable and after full recovery returned to the ward.

Results of arterial blood gas analyzes BUN, AST, ALT, LDH, glycemia, total bilirubin, proteinogram, haemogram and electrolytes were within normal range reference. The patient's postoperative course was uneventful and she was discharged home on the third postoperative day.

\section{Discussion}

Dextrocardia with situs inversus totalis without Kartagener syndrome is a rare condition, 1 out of 5 patients with situs inversus has Kartagener syndrome. Patients with Kartagener syndrome usually present very different problems due to multiple structural and functional abnormalities that coexist with it [10].

A long time ago, dextrocardia was diagnosed only at autopsy, but now thanks to improvements of diagnostic technology like $\mathrm{X}$ - ray, EKG, echocardiography, CT, ultrasound and MRI, it can easily be detected. The care should be taken to chest radiography due to the errors that might occur over labeling. This is a common cause of a false- negative diagnosis of dextrocardia such it was in our case. When dextrocardia is presented with situs inversus, the heart is frequently normal [11]. The incidence of congenital heart disease is very low- about $3 \%$, whereas incidence of Kartagener's syndrome will occur in about $20 \%$ of these patients [12].

Most patients with these syndromes were detected in infancy or childhood due to these associated anomalies, although some of them remained undiagnosed until adulthood [13]. Our patient was previously healthy and did 
not know anything about her condition, but the diagnosis proved our suspicions.

The anesthetic implications of dextrocardia with situs inversus totalis are different. Heart rate, arterial blood pressure and endtidal concentration of the $\mathrm{CO}_{2}$, were within the physiological ranges intraoperatively. The recovery was smooth with no agitation. Because of some theories that volatile anesthetic concentrations may be too low and cause awareness, patient was interviewed postoperatively in the postanesthesia care unit (PACU) and denies having remembered anything during the operation.

There was no impact of low flow anesthesia and patient condition in gas exchange as was shown by arterial blood gas values $-\mathrm{PaO}_{2}$, $\mathrm{PaCO}_{2}$ and $\mathrm{pH}$ did not change over the course of anesthesia and 2 hours postoperatively.

Kharasch et al. reported no significant differences, between the hepatic and renal effects in patients undergoing longduration low-flow sevoflurane anesthesia [14]. In our case low-flow sevoflurane anesthesia has not shown to have any effect on hepatocellular function, as measured by AST, ALT, bilirubin and proteinogram. Renal function was evaluated by using standard clinical measures of renal function (BUN, serum creatinine, and creatinine clearance), we did not find any specific effect of lowflowsevoflurane on renal function which corresponds with other investigations [1520].

\section{Conclusion}

With particular attention and vigilance in preanesthetic visit we managed to find out dextrocardia with situs inversus totalis. We realized low flow sevoflurane anesthesia in this patient, which showed to be safe, and her intra and postoperative course was uneventful. She was discharged home on the third postoperative day in good health.

\section{References}

1. Cleveland M. Situs inversus viscerum: anatomic study. Arch Surg 1926; 13:343.

2. Fuster V, Alexander RW, O'Rourke RA, et al, eds. Hurst's the Heart. 10th ed. New York, NY: McGraw- Hill; 2001.

3. Bajwa SS, Kulshrestha A, Kaur J, Gupta S, Singh A, Parmar SS. The challenging aspects and successful anaesthetic management in a case of situs inversus totalis. Indian J Anaesth. 2012; 56(3): 295-297.

4. J. Singh, I. Muntyan, Y. Fulman: A patient with Situs Inversus without ciliary dysfunction presenting for urgent Cesarean Section: considerations for a safe anesthetic. The Internet Journal of Anesthesiology. 2008 Volume 16 Number 2.

5. Cohen IT, Hannallah RS, Hummer KA. The incidence of emergence agitation associated with desflurane anesthesia in children is reduced by fentanyl. Anesth Analg 2001; 93: 88-91.

6. Steward DJ. A simplified scoring system for the postoperative recovery room. Can J Anaesth 1975; 22:111-3.

7. Brice DD, Hetherington RR, Utting JE. A simple study of awareness and dreaming during anesthesia. Br J Anaesth 1970; 42: 535-41.

8. Sandin RH, Enlund G, Samuelsson P, Lennmarken C. Awareness during anaesthesia: a prospective case study. Lancet 2000; 355: 707-11

9. Liu WHD, Thorp TA, Graham SG, Aitkenhead AR. Incidence of awareness with recall during general anaesthesia. Anaesth 1991; 46: 435-7.

10. Winer- Muram HT. Adult presentation of heterotaxic syndromes and related complexes. J Thorax Imaging 1995; 10(1): 43- 57.

11. J. Reidy , S. Sischy , V. Barrow , Anaesthesia for Kartagener's syndrome Br. J. Anaesth. (2000) 85 (6): 919- 921. 
12. Nugent EW. The pathology, abnormal physiology, clinical recognition, and medical and surgical treatment of congenital heart disease. In: Hurst, Ed. The Heart. 7th Edn. New York:

13. Ohara N, Teramoto K. Situs inversus with dextrocardia diagnosed in the third trimester. J Obstet Gynaecol 2002; 22(3):317- 318

14. Kharasch ED, Frink ED Jr, Artru A, et al. Long-duration low-flow sevoflurane and isoflurane effects on postoperative renal and hepatic function. Anesth

Analg 2001; 93: 1511-20.

15. Mazze RI, Callan CM, Galvez ST, et al. The effects of sevoflurane on serum creatinine and blood urea nitrogen concentrations: a retrospective, twenty-two-center, comparative evaluation of renal function in adult surgical patients. Anesth Analg 2000; 90: 683-8

16. Bito $\mathrm{H}$, Ikeda K. Renal and hepatic function in surgical patients after low-flow sevoflurane or isoflurane anesthesia. Anesth Analg 1996; 82: 173-6.

17. Bito H, Ikeuchi Y, Ikeda K. Effects of lowflow sevoflurane anesthesia on renal function: comparison with high-flow sevoflurane anesthesia and low-flow isoflurane anesthesia. Anesthesiology 1997; 86: $1231-7$

18. Bito H, Ikeda K. Closed-circuit anesthesia with sevoflurane in humans: effects on renal and hepatic function and concentrations of breakdown products with soda lime in the circuit. Anesthesiology 1994; 80: 71-6

19. Kharasch ED, Frink EJ Jr Zager R, et al. Assessment of low-flow sevoflurane and isoflurane effects on renal function using sensitive markers of tubular toxicity. Anesthesiology 1997; 86: 1238-53

20. Higuchi H, Sumita S, Wada H, et al. Effects of sevoflurane and isoflurane on renal function and on possible markers of nephrotoxicity. Anesthesiology 1998; 89: 307-22. 